\title{
To the theory and practice of psychosomatics
}

\author{
Alexander V. Khaikin \\ Training center "The practices of self healing", Rostov-on-Don, Russia
}

Accepted 20 June 2012

Original Russian Text (C) Khaikin AV, 2012, published in Saratov Journal of Medical Scientific Research 2012; 8(2): $193-198$.

\begin{abstract}
The author formulates the main topical theoretical issues of psychosomatics. It provides an overview of psychosomatogenesis models. It gives the hypothesis of the author about psycho-biological nature of cancer and mechanism of the placebo effect. It formulates a general model of psychosomatic work. It provides a brief overview of psychotherapeutic techniques used in the work with the somatic diseases, where the main attention is paid to the procedure and hypnotherapeutic techniques. Description of the author's techniques. It discusses the question of the integration of psychosomatics and somatic medicine.
\end{abstract}

Keywords: psychosomatics, psychoneuroimmunology, self-healing, placebo-effect, cancerogenesis, oncology, self-regulation.

Cite as Khaikin AV. To the theory and practice of psychosomatics. Russian Open Medical Journal 2012; 1: 0104.

Correspondence to Alexander V. Khaikin. Address: 11, per. Universitetsky, Rostov-on-Don, 344022, Russia. Phone: +7-(903)-401-8210, +7-(863)-2218210.

Current status of relationship between somatic medicine and an oriented to the work with somatic area of psychotherapy (psychology) can be largely associated with confrontation than cooperation. From the one hand, there are undeniable successes of medicine which based on findings of such «materialistic» sciences as physics, biomechanics, biochemistry, microbiology, physiology etc., has learned to effectively fight against various human body diseases. Despite clear declining «rates of effectiveness growth" in the recent decades, the medicine in general feels self-sufficient in the theory and practice of medical treatment. On the other hand, developing psychosomatic methods give us a fair number of healings from various diseases even considered as incurable at the state-on-the-art level of medical science $[1,2,3]$. Moreover, psychological "treatment" does not involve any adverse side effects as pharmacological treatment.

However, psychosomatic methods are rather new and they haven't gained the same widespread acceptance as the medical ones yet. Besides, these methods do not always demonstrate stable results. But subject to the further development of their technologies and growing popularity, the psychosomatic methods, to the opinion of their numerous supporters, can become an alternative to medical treatment. Therefore, both approaches "have something to put together». Possessing the developed methodologies of comprehensive investigation of a common subject and having combined their efforts, together they more likely can flush out any blind spots in understanding any disease genesis as well as the ways of their handling.

Obviously, for the further development, psychosomatics has to examine a number of fundamental theoretical questions. And the first question which divides the medics and psychologists but makes their efforts in finding an answer to which they can combine their research resources, is the question about «position, relation and interaction of psychic, physiologic and material factors in genesis of human body diseases». Certainly, a concept of a role of the psychic factor in genesis of human body diseases always depends on general ideas of a role and position of psychics in the material world prevailing in each epoch; and history of their development can be represented as a turn of dialectical spiral. In the times of panpsychism, while understanding nature of diseases and methods of their healing psychological factors were decisive. Besides, traditional Chinese, ancient Greek and medieval Arab medicines were based on an idea of integrity of the material and the psychic understanding that "one shall cure a human but not a disease". However, subsequently, prevailing in Europe Cartesian ideology and rapid development of natural sciences (along with some "delay» in psychology development) resulted in more and more "materialistic" medicine. Twist of fate: medicine started "clearing from any psychic matter» just at the same time when the European scientific psychology began forming. It should be noted, that after assuming a human or, more exactly, a human body as biomechanical, biochemical and electrical machine, European medicine has managed to make a huge theoretical step forth in its development, results of which have been used up to date. Nevertheless, the Newton-Cartesian worldview crucially dividing matter and conscience and considering a human body (as well as the whole world) as a machine, has begun to give way to ideas of more integrity and indivisibility of matter and conscience thanks to, first of all, development of the modern quantum-relativistic physics. And at the same time, development of psychology and psychotherapy has resulted in formation and strengthening of psychosomatic concepts. In the end of a spiral turn, a new view has formed and strengthened which considered phenomena of emotional life, inner psychical conflict and unrealized human wants as main causes of body diseases. It has been suggested that just a.m. factors lead to pathophysiological processes through activity of brains, nervous system and then through the regulated by it systems of endocrine control, immunity, digestion, blood supply etc. Moreover, it has been raised an issue on 
correspondence of different psychological types with certain medical problems [4].

Hereby, investigation of the matter under discussion can be represented in the following models:

a) psychics - the cause that triggers «incorrect» functioning of a human organism, somatic pathology which by now can develop independently from its original cause as well as continuously demands for «management»from the part of psychic matter;

b) mental conflicts and any pathological somatic process are not a cause and an effect but the elements of some psycho-material integrity, therefore, formulation of the question on psychic factors as a cause shall be incorrect;

c) psychic phenomena are the factors that only facilitate any pathological somatic process by speeding it up or inhibiting.

In recent decades, the arguments in favor of the determining connection between psychics and somatic health or disorder with processes of a disease onset and recovery, have been complemented with the results of observations of patients suffering multiple personality disorder. They have revealed that in the moment of transition from one personality to another, allergy, diabetes and even (!) neoplastic process can disappear so fast that can not be explained neither by modern physiology nor by pathophysiology [3]. On the other part, supporters of necessary participation of psychological conflict in genesis of any physical disease have to answer multiple questions-objections, e.g. how such opinion correlates with the facts of animals and plants disease incidences.

Hereafter, if after all we acknowledge psychic factor as a cause, then another question would be actualized «How does any unresolved inner mental conflict and unsatisfied psychological needs transform into somatic disease?».

In psychotherapeutic and psychological literature there are a number of various models with description of psychosomatic genesis. In the author's opinion, they can be classified into ten main models which are represented below without reference to particular psychotherapeutic systems and particular authors.

"Symbolic" model is predicated on the assumption that repressed needs and conflicts "try" to come up through a disease symbolizing the repressed ones. Symptoms of disease are analogous to unrealized, blocked emotions and actions. For example, unshed tears can be expressed through a running nose. The following five models consider a disease as an effect of mental conflict. "Energy-asthenic» model proposes the excess consumption of vital energy in attempts to resolve intrapersonal conflict and the resulting shortage of energy for fighting physical disease-producing factors to be a cause of a disease.

"Energy-dynamic" model considers somatogenesis as follows: psychic energy of unrealized needs and wishes seeks and finds a pathological, alternate way for their outcome, namely, to organs and body parts, making them disordered. This model brings together perceptions of European psychosomatics and Chinese medicine.

Model "deficit of impressions" is based on a hypothesis that emotional impressions (especially positive ones) in a nervous system transform into some substances necessary for a human to the same extent as vitamins. Those humans, which can not live "hic et nunc" because of their intra conflicts, receive less emotional impressions and, consequently, become ill.
"Physiological» or "stress» model tries to determine the mechanism of transformation of any intra mental conflict into medical diagnosis in more details. "Normally», a human satisfies his actual need or refuses (temporally or entirely) from its satisfaction. However, by virtue of his inner non-identity, instead of that, often he unconsciously keeps trying to satisfy any repressed need. In turn, process of need satisfaction is connected with emotions which represent the whole reaction of psychics and an organism itself. Emotional reactions include activation or suppression of such important physiological processes as blood pressure, digestion, immunity, internal secretion etc. And these processes being switched "on" and "off" for an abnormally long period of time, naturally result in human disorders.

"Retroflex" model is predicated on the assumption that, by way of rejection from satisfaction of any "prohibited" need, a human unconsciously chooses refocusing actions for its satisfaction which should be focused on the external world, on himself (and consequently, on organs and system of his body).

In the "functional" or "secondary benefit" model, a disease is not considered as consequence of intrapersonal conflict and nonsatisfaction of any need but as a compromise between any conflicting sides and a "childish", "pathological" method of their needs satisfaction. This method has been applied since our childhood, when our disorders helped us to organize actions and feeling of other people to us in a proper way. Thus, any disease has its function and sense and, moreover, often in any «transpersonal» system, e.g. in a family.

Model of "cleaning" also supposes that any disease has its function and, as well as the following model, can be deemed as a particular case of the previous one. In this case, a disease seems trying to destroy, "amputate» that part of a human body which has been "violated» (e.g., in case of sexual assault), or to "clean» the whole organism and a soul having punished it with suffering. .

"Thanatical" model: a disease carries out slow suicide, whereby a need standing behind any strive for death can have various natures.

"Process" or "development" model considers a disease not as a pathology but as a stopped process of self-identity development. Any disease itself comprises a remedy for recovery. This position is close to the Oriental esoteric teaching which deems diseases as a source and stimulus of the spiritual development.

As we can see, some models of psychosomatic genesis describe it as a consequence of inner mental conflict, and other models provide disease with some useful functions realized by a rather non-useful way. The question about an extent to which the above mentioned models represent complementary descriptions of a single process "at different angels", where in every model different aspects of a single mechanism can be distinguished, as well as about an extent to which these different models represent descriptions of genesis of different nosologies, should be further analyzed. In general, it should be noted that not many models keep trying to describe the mechanism of transformation of psychological problems into pathological physiologic processes. Nevertheless, a positive effect of each model is an indication on a possible way of psychotherapeutic interference in psychological causes of any somatic disease. Certainly, every model "gravitates» toward a particular psychotherapeutic system. Besides, there still remains one disputable question whether it is expedient to associate some certain nosologies with particular types of psychological problems. This point of view is evidently adequate to 
the research purposes but in any particular therapeutic case a completely individual approach is also important.

Solution to the first question about a role and position of psychics in any disease genesis also has an effect on the question "Which of physical human diseases can be referred to the psychosomatic ones?». And in this connection, it's not clear until now - to which extent infectious diseases or pathologies of newborn babies can be attributed to psychosomatic? In the medical community even those specialists committed to psychosomatic ideas still debate whether it is possible to consider oncology as psychosomatic.

Within the framework of the last question, the author thinks to be appropriate his hypothesis of psychological or, more precisely, psycho-biological mechanism of oncological diseases contraction. It is known that unicellular organisms are not subject to ageing - apoptosis - in a way as multi-cellular organisms do. (Apparently, apoptosis is somewhat like "compensation» for the high externally influenced adaptability and survivability inherent to multi-cellular organisms and non-available for unicellular organisms; in this way nature blocks up possible overpopulation and stoppage of evolutional growth). Atypical cancer cells are not subject to apoptosis as well. And outgrowth of atypical cells number as well as insufficiently active immunity "supporting» this process can be deemed as protest or "rebellion» on a deeppsychological, psychobiological level against inevitable ageing and dying of old age. Attempt to replace «mortal» cells by «immortal» ones. Nevertheless, under the known reasons, this attempt to get immortality leads to an adverse effect - even more early death of the organism. Apparently, a part of psychics and organism in whole, which are responsible for this process, do not know about its consequences. On the basis of this hypothesis, one can draw up a strategy of psychotherapeutic (psychological) work. For example, in hypnotic-therapeutic approach one can apply metaphors, metaphoric stories describing the above mentioned scenario. (Of course, the author does not mean absence of any other possible psychological causes in oncology genesis). Besides, the author takes risk to suppose that development of such view on oncology nature in the future can lead to application of this powerful oncology mechanism as well as its known behavior for the purposes of the organism rejuvenation.

Question on a possible role and position of the psychics in a human body healing is connected with the corresponding question on disease genesis, however, only partially. Hence, it has actually the same history. Even ancient physicians knew that "winners' wounds heal faster». However, the concept that positive emotions facilitate recovery and maintain health and negative emotions result in disease and slower recovery, has turned out to be not correct. Important: not positive or negative emotions do matter but the fact whether they stimulate or depress the searching activity [5]. Just the searching activity, to the opinion of Rotenberg V. S. and Arshavsky V. V., is the major factor influencing a degree of human organism resistance to any disease-producing factors.

Besides, ancient physicians knew that «that doctor is bad if a sick man does not feel better looking at him». Any doctor, inter alia, shall instill confidence in healing or pending improvement. This moment is naturally connected to a number of events directly related to our discussed issue. Such events as spontaneous remission and placebo effect are well-known to medicine. Please note, that independently of the fact, whether we consider psychological or physical factors to be a cause of disease, whether we believe that self-healing mechanism has just physiological nature, nevertheless, we must admit that "starting method" of placebo effect is purely psychological. And in this aspect a question about a role of psychics in self-healing does not depend on a role of psychics in a disease genesis.

Notwithstanding the well-known factors of spontaneous remission and healing thanks to placebo effect even from those diseases which can not be treated by the modern medicine, and therefore apparent importance and potential of usage of these super effective healing mechanisms, there is still no answer to the question "On which inner psychological and physiological systems are the self-healing process based in cases of spontaneous remission and "placebo effect? ». Widely known (not in medicine) thesis "faith cures", to the author's opinion, is simply a redefinition of the problem. We still have to find answer to the following questions: how does the faith cure? by means of which mechanisms? by stimulation of which conditions or ways of functioning etc?.

Here the author believes to be useful his own hypothesis, possibly, "bringing light" to the placebo essence. One of the questions correlated with researching this theme and representing serious interest, to the author's opinion- is the question "what for, being completely sure in healing effect of an external remedy (placebo), a human unintentionally triggers healing powers of his organism and psychics? ". Possible answer is as follows. Healing mechanisms can be classified into "static» and "dynamic». "Static» are more focused on blockade of any disorder process («to prevent deterioration»), e.g. inflammation. Herewith, during their work "static» mechanisms block up not only propagation and progression of disease. Global startup of the stopping "static» process also blocks working of the "dynamical» process. "Dynamic" mechanisms (and "supereffective» relate just to them) are intended to "feeling better», they seems rather generating health than fighting against disease. One more outstanding feature - they work irrespective to different conditions. On the contrary, «static» mechanism, just after being informed that there is a remedy which guarantees full recovery, is slowing down, thereby unbraking "dynamic» thus resulting in the effect of spontaneous remission. (Certainly, the author realizes that the above mentioned hypothesis is rather an issue for further investigation than a finished scheme).

Obviously, the next question: «If there are any methods of voluntary startup of the supereffective self-healing mechanisms and, if yes, then what are they? ". (Apparently, in the practical aspect modern hypnotherapy will be the closest to solution of this problem). Besides, the more extended question will be of importance: "What are the possible perspectives of effectiveness development and a scope of application of psychological (psychotherapeutic) methods in physical diseases healing? ").

On the basis of the above and of the well-known practice of psychosomatic work, the author proposes to describe the latter as a solution to three tasks as follows:

1) Preparation of a client (patient) to the major work, namely:

a) to ascertain the client's concept of disease and draw the client's and therapist's concepts together;

b) to work with motivation for recovery;

c) to work with personal reaction for disease;

d) to create belief in the possibility of recovery;

e) to recover energy potential of the client up to a level required for the main work conducting; 
2) Working-out intrapersonal conflicts which cause disease and reviving integrity;

3) Activation of the inner healing powers of any organism and psychics, focusing them on fighting against disease.

It shall be noted that mass media, popular literature and experience of any previously cured patients can popularize the idea that physical diseases can be caused by unresolved psychological problems, that there are "powerful" internal healing reserves and that physical disease can be cured in result of work with a psychologist (psychotherapist). If a human with somatic diagnosis has come to a psychotherapist, it means that he/she already informed. However, it's very important to remember that in his/her original ideas such client can qualify:

a) disease - as an enemy and process of healing - as a struggle;

b) disease - as failure or shortage of something and healing - as refit or concern;

c) disease - as due punishment;

d) disease - as an enemy which is impossible to defeat but possible to negotiate or take its strategy;

e) disease - as a result of improper life style and unrealized psychological needs or as an ally or teacher and to the healing process - as learning, development, recovery not only physical but psychical, finding ways of changing the whole life.

Besides, to a greater or lesser extent, in the process of healing the client may be ready to rest upon himself, to believe that here the decisive role pertains to a specialist or to the highest powers. All the above considerably define a strategy of preliminary work, from the one side, and from another side, can be used on preparation stage as well as while choosing means and contexts in the process of the main work.

In order to resolve the first task of psychosomatic work, especially for the work with confidence building, all techniques for manipulation with persuasions including technique of neurolinguistic programming (NLP) and "eye movement desensitization" technique (Shapiro F.) [6], are appropriate. Besides, in case of actual astenisation of a client and required preliminary work for initial revival of energy potential, a number of hypnotherapeutic techniques can be applied.

For resolving the second task, techniques of various approaches of personal and family therapy to be applied in compliance with any particular model of psychosomatic genesis used in each approach. However, to the author's opinion, the process approach techniques are the most advanced here.

To the process approach techniques we can refer «ideodynamic techniques» by Rossi E. [7,8], «focusing» by E. Gendlin [8,9], «epistemological metaphor» by D.Grove [8], «process work» by A. Mindell $[10,11]$, «Hakomi» by R. Kurtz [12] and "somatic experiencing» by P. Levin [13]. The author believes that in psychotherapy the basis for the process techniques development will be the principles of utilization and pacing\&leading applied in hypnotherapy by M. Erickson and his followers [14], as well as making more importance to the therapy process regarding its content in gestalt-approach [15]. Consideration of a disease as the stopped, but trying to continue, development and invention of integrity are common for every process approaches. Therefore, the main therapeutic resources here shall be searched in symptoms of a disease. An object of the therapist is supposed to be creating conditions for "reversing» symptoms into curative process. Without forcing any content upon a patient and without violation to the process, the therapist only follows the process of disclosure of essence, symptoms and their transformation into the greater integrity. Nevertheless, having much in common, these process approaches demonstrate great distinctions in the form of process leading. All approaches substantially rest on working with physical experience, but only Hakomi and somatic experiencing can be referred to the methods of body-oriented therapy. In his work D. Grove places primary importance on visual metaphors of body experience. In Mindell's therapy, equal importance is allotted to all «channels». Besides, the methods by D. Grove and P. Levin as distinct from the others are entirely focused on working with consequences of any psychological traumatic experience. Although in every process approach, clearly or actually, great attention is paid to creation of a permitting, interested and friendly "observer" or "metacommunicator», extent and effect of disengagement, noninvolvement, dissociation of an "observer» from any observed experience in different approaches essentially differs. Thus, in process work by A. Mindell, on the contrary, effect of association and embodiment (as in gestalt-therapy) is very important. Especially important is dissociation of an observer from any observed process in idiodynamic techniques by $\mathrm{E}$. Rossi, in approaches by D. Grove and E. Gendlin. These three approaches are oriented to trance induction (focusing - under definite method of leading) and, to the author's opinion, can comprise a unified trend - «procedural hypnotherapy».

As an illustration to procedural technique, the authors considers appropriate to describe experience of self-healing against influenza, which further has become step-by-step technique in the style of process working by A. Mindell.

Having a high fever and sensation of burst in his body as well as respiratory symptoms, the author began moving around the room, simultaneously affording himself to feel soreness in his body to the full extent. Both strength of these feelings and tendency to bursting easily allowed him to "expand» these feelings all over the room as if they created in the room some kind of a "field»; and it was the following step. On the next step, perception of such "field of feeling", at the moment, from outside, gave the author some "hints" on how to move in the most energy-saving way "quite natural" in this "field", and he began to move in such a way. Having moved during some time "adjusting" his movements, the author proceeded with the next step, namely, asked himself: "Who does move in such a way like I do right now?». And keeping moving, after a while he felt himself like a Siberian tiger wearily making its way through the snow-covered taiga, slumping in deep soft snow. And keeping moving and feeling himself a tiger, i.e. a figure representing essence of the symptom "field of feeling", the author, having experienced quite a new self-perception, found out that the symptoms of disease actually disappeared. All of this took approximately 20 minutes. Now, after more careful investigation of the "process work", the author has found appropriate to add to this technique two more Mindell steps, namely, answers to the questions: "What is a message of a figure of any symptom field (experience) and what can it introduce into my life?", and "What namely does (or did) prevent to integrate it into my life?». Answer to the last question can lead to the further stage of work. This technique, named by the author "Movement in the field of symptoms", can be used not only in psychosomatic work but also in the frames of the work with any strong, "bursting outward» emotions, psychic tension, angst, but even, if limited by stage of seeking and movement, corresponding to the "field" emotions. 
For resolving to second task, hypnotherapeutic technique of step-by-step reformation [16] where any work is resolved to searching and replacement in trance of any unacceptable method of reaching an important target or need, i.e. disease, to the found new method, has also proved to be rather effective.

For the purposes of the third task resolving, it should be underlined that successes in development and utilization of principle of random access to these healing human body resources and unconscious part of psychics, are generally related to the techniques of working in trance states. In such work one can use direct and indirect suggestions which can be verbal [7] or representing visualizations [1]. Often in such healing work one applies verbal and visual metaphors of disease, disease-producing factors, healing resources, process of recovery itself as well as "inner healer"." Such work may be carried out by a patient as a self-maintained exercise or followed-up by a hypnotherapist. Trance can be introduced preliminary, but similar visualization itself facilitates trance induction.

For illustrative purposes we'll take description of the author's technique "Three screens» which has been developed by him for working with "visual» clients and represents therapeutic suggestion - a visual metaphor. Together with preliminary introducing (self-introducing) of shallow- or middle-depth trance, a client is suggested to visualize three monitors on a desktop or one monitor where, besides the main one, there are two additional smaller-size monitors, e.g. in the upper corners. The first and the third monitors are equipped only with a "push" button, the second (in the middle) is equipped with a special keyboard with handles and triggers. On the first screen (monitor) the client visualizes his unhealthy organ as he imagines it (actually, a real X-ray photograph would be good enough), on the third monitor the same organ but after treatment. Then, having visualized the unhealthy organ on the second monitor, he starts experimenting with the keyboard elements experiencing with their "functional purpose» (according to changes on the second monitor) and, in such a way, trying to transform an image of the unhealthy organ into a healthy one. It would be good, if the client will add to the image his perceptions of pushbuttons, handles, triggers as well as sounds, because in will intensify reality and deepen trance.

If this technique is used in self-study, then it is recommended to repeat the technique as an everyday practice. Moreover, possibly it will be required to find out a new algorithm every day.

Besides, application of the process hypnotherapeutic technique by $\mathrm{E}$. Rossi where trance is induced not while entering into state of relaxation and calmness as in most visualizing techniques but, on the contrary, at the peak on excitement and tension, would be very effective in resolving this problem.

The techniques of neuro-linguistic programming are also very similar to hypnosis methods. Among them, to the author's opinion, the most interested is the technique by $\mathrm{K}$. Andreas "application of natural physical capability for healing», where in his wok the client uses transfer of self-healing experience and, very important, uses transfer of experience of relation to selfhealing possibility from one disease to another [2].

Techniques of direct psycho-energetic self-regulation of physiological processes (omitting the unconscious) also have proved their effectiveness. "Bioenergtraining" by Vasyutin A.M. can serve as example of such method [17].

Of course, resolving different tasks of psychosomatic work does not always mean its stages. This mainly concerns the second and the third tasks. Often they are resolved simultaneously and by the same methods. Apart form the main three tasks, a task of working with pain and a task of emotional self-regulation as well as teaching techniques of stress coping as one of the ways of body diseases prevention, are also crucial.

Turning back to the discussed in the beginning of the article issue on integration of psychosomatic work with medicine, it can be stated as follows. Presently, psychosomatics has possibility as to become an interdisciplinary approach, so to continue its development as an alternative to medical approach. As a positive example of really emerged tendency towards interdisciplinary approach one can consider psychoneuroimmunology and mind\&body medicine. The new trend, which to the great extent has appeared thanks to the discovery by Ader R. on association of psyche and nervous system with immune system previously considered to be rather autonomous, encouraged by achievements of the married couple of Simontons and works by Siegel B. $[2,18]$, can become a basis for the further cooperation and paradigms closing. Obviously, integration of resources may be possible not only in investigation of theoretical issues but creating organizational forms for the joint practical work. (The author has his own experience of working in such medical center where doctors and psychologists on equal terms are engaged in healing humans from chronic diseases; had an opportunity to see the highest effectiveness of such cooperation.) However, to the author's opinion, any integration both in theoretical and practical activity can take place only subject to rejection by each party of their conviction in "exclusive possession» of the truth. Presently, the fulfillment of such condition itself is a non-trivial issue.

\section{Reference}

1. Simonton K, Simonton S. Cancer Therapy. St. Petersburg, 2001: 288 p.

2. Andreas $K$, Andreas S. The heart of the mind. Novosibirsk: ECORA, 1995: $375 \mathrm{p}$.

3. Talbot M. The holographic universe. Kiev: Sofia, 2004: 366 p.

4. Alexander F. Psychosomatic Medicine. M.: Penguin Books, 2002: 351 p.

5. Rotenberg VS, Arshavsky VV. Search activity and adaptation. Moscow: Nauka, 1984: 192 p.

6. Kovalev SV. Healing with NLP. M, 1999: 576 p.

7. Becchio J, Rossi E. Hypnosis 21st century. M.: Class, 2003: 263 p.

8. Khaikin AV. Procedural methods after Erikson hypnotherapy in work with crisis states. Psychology of crisis and crisis states. Proceedings of the International Interdisciplinary Symposium. Rostov-on-Don, 2007: 121-125.

9. Dzhendlin U. Focusing. A new therapeutic method of dealing with feelings. M.: Class, 2000: $448 p$

10. Mindell A. On the edge of life and death. M.: Class, 2000: 256 p.

11. Mindell A, Mindell E. Gallop, back to front: Process work in theory and practice. M.: Class, 1999: $224 \mathrm{p}$.

12. Kurtz R. Body-oriented psychotherapy. The method of Hakomi. M.: Class, 2004: $304 \mathrm{p}$.

13. Levine $P$, Frederick E. Waking the Tiger - healing trauma. Moscow: AST, 2007: 316 p.

14. Gaudin J. The new hypnosis. Introduction to Ericson hypnotherapy. M: From the Institute of Psychotherapy, 2003: 298 p.

15. Gestalt therapy. Theory and practice. M: EKSMO, 2001: 320 p.

16. Bandler R, Grinder D. Transformation. Syktyvkar: Flinta, 1994: 296 p.

17. Vasyutin AM. Psychological ginseng. Effective self-regulation techniques. Rostov-on-Don, Phoenix, 2004: 288 p.

18. Defeat yours illnesses. / Ed. D. Golmen, D. Gurin. Mn.: Popourri, 1999: $640 \mathrm{p}$. 
Authors:

Alexander V. Khaikin - PhD, Chief of Training center «The practices of self healing», Rostov-on-Don, Russia. 